\title{
Elevated Red Cell Distribution Width as a Prognostic Marker in Severe Sepsis: A Prospective Observational Study
}

\author{
Aditya Jandial, Susheel Kumar, Ashish Bhalla, Navneet Sharma, Neelam Varma'1, Subhash Varma \\ Departments of Internal Medicine and ${ }^{1}$ Hematology, Postgraduate Institute of Medical Education and Research, Chandigarh, India
}

\section{Abstract}

Introduction: Sepsis is a dysregulated host response to infection resulting in potentially life-threatening organ dysfunction. Elevation in red cell distribution width (RDW), a simple routinely done investigation, could be a prognostic marker in these patients. Methods: Between January 2014 and June 2015, 200 patients with severe sepsis at admission were prospectively evaluated for association between RDW at admission and 30-day mortality. Besides the groups of raised and normal RDW, study population was further analyzed after categorizing into three RDW groups as follows: $\leq 14.5 \%, 14.6-17.3 \%$, and $>17.3 \%$ as well. To find out factors associated independently with 30 -day mortality, we applied multivariate logistic regression analysis. Results: Among 200 patients, 115 (57.5\%) were males. Mean age of the study subjects was 51.32 \pm 16.98 years. Mean RDW at admission was $17.40 \pm 3.21 \%$, ranging from $12.6 \%$ to $33.3 \%$. Mean Acute Physiology and Chronic Health Evaluation II (APACHE II) score of study population at admission was $22.49 \pm 5.72$. One hundred and fourteen (57\%) patients had 30-day mortality. Even though RDW showed a hierarchical association with 30-day mortality among three RDW groups, it was not found to be an independent predictor of 30-day mortality. APACHE II score, serum albumin, partial pressure of arterial oxygen/fraction of inspired oxygen ratio, and serum fibrinogen level at admission were observed to be independent predictors of 30-day mortality. Conclusions: In severe sepsis patients, RDW though showed a graded relationship with 30-day mortality was not found to be an independent predictor of 30-day mortality.

Keywords: 30-day mortality, Acute Physiology and Chronic Health Evaluation II score, red cell distribution width, severe sepsis

\section{INTRODUCTION}

Sepsis is a life-threatening clinical condition that has cost humanity heavily since time immemorial. This clinical syndrome has been found to have increasing incidence throughout the world in recent decades. ${ }^{[1,2]}$ Initially, sepsis was defined as systemic inflammatory response to infection, noting that multiple noninfectious causes could elicit the similar response..$^{[1-3]}$ In 2001, a second consensus panel expanded the list of variables for defining sepsis including organ dysfunction parameters as well. ${ }^{[4]}$ Severity of illness and mortality risk escalates with severity of organ dysfunction. Severe sepsis and septic shock carry high potential mortality rates, possibly up to $40 \%-50 \% .{ }^{[2]}$ Prognostication in severe sepsis may facilitate aggressive management of particular patient groups. Prognostic factors such as age, sex, comorbidities, biomarkers (C-reactive protein [CRP], procalcitonin, etc.), and severity of illness score (Acute Physiology and Chronic Health Evaluation [APACHE], etc.,) have been reported to be associated with the outcome in cases of severe sepsis. ${ }^{[5-7]}$

\begin{tabular}{|l|l|}
\hline \multicolumn{2}{|c|}{ Access this article online } \\
\hline Quick Response Code: & Website: \\
\hline & www.ijccm.org \\
\hline & \\
\hline
\end{tabular}

Red cell distribution width (RDW) represents the variation in size of all the red blood cells (RBCs) in an individual patient. It is elevated when excess of reticulocytes are released into the circulation. Over and above its role in the evaluation of anemia, RDW has been found to be an important prognostic marker in the patients with cardiovascular disorders, pulmonary embolism, community-acquired pneumonia, and critical illness. ${ }^{[8-11]}$ The association was independent of covariates such as nutritional status, anemia, other inflammatory markers, and comorbidities. Inflammation and oxidative stress have been suggested to reduce RBC survival and suppress their maturation resulting in release of large premature RBCs into circulation, contributing to elevated RDW. ${ }^{[11-13]}$ Inflammation

Address for correspondence: Dr. Susheel Kumar, Department of Internal Medicine, Postgraduate Institute of Medical Education and Research, Chandigarh - 160 012, India. E-mail: sk_bhutungru1977@yahoo.co.in

This is an open access article distributed under the terms of the Creative Commons Attribution-NonCommercial-ShareAlike 3.0 License, which allows others to remix, tweak, and build upon the work non-commercially, as long as the author is credited and the new creations are licensed under the identical terms.

For reprints contact: reprints@medknow.com

How to cite this article: Jandial A, Kumar S, Bhalla A, Sharma N, Varma N, Varma S. Elevated red cell distribution width as a prognostic marker in severe sepsis: A prospective observational study. Indian J Crit Care Med 2017;21:552-62. 
and oxidative stress are the essential components of sepsis cascade. ${ }^{[2]}$ Complete blood count $(\mathrm{CBC})$ is nowadays done in most of the sepsis patients admitted to the emergency medical services by automated analyzers all over the world. RDW is routinely provided within the $\mathrm{CBC}$ done by automated analyzers. Inexpensive, routinely available, and rapidly measurable prognostic tools have clinical utility in the identification of subset of patients with severe sepsis who need aggressive management. RDW could be a useful tool in prognostication of cases with severe sepsis as described in recent studies. ${ }^{[14-16]}$ Contemplating above points, we performed this prospective observational study to assess role of elevated RDW in predicting 30-day mortality in patients with severe sepsis presenting and getting admitted in emergency services of a large referral hospital in North India.

\section{Materials and Methods}

It was a single-center prospective observational study undertaken in emergency medical services of a tertiary care institution in North India from January 2014 to June 2015. Two hundred adult ( $\geq 18$ years old) patients with diagnosis of severe sepsis for $>24 \mathrm{~h}$ were enrolled in the study. Severe sepsis was defined according to the 2003 statement. ${ }^{[4]}$ We excluded patients who denied formal consent, pregnant females, had history of packed RBCs transfusion in the previous week, known hematological disorders (leukemia, myelodysplastic syndrome, neoplastic metastases to bone marrow), recent chemotherapy, immunosuppression for solid organ transplantation, postsplenectomy, use of drugs known to induce changes in the morphology, and rheology of RBC (erythropoietin, pentoxifylline, cyclosporine, nitrates, etc.). All the patients were managed according to the Surviving Sepsis Campaign 2013 Guidelines. ${ }^{[2]}$

Standardized data collection forms were used to collect the information pertaining to demographic, clinical, and laboratory parameters of the study subjects. Demographic variables included age, sex, and religion. Clinical variables included primary site of sepsis, pulse rate, blood pressure, respiratory rate, temperature, and Glasgow coma scale (GCS). APACHE II score as a measure of severity of illness was calculated using the values of clinical and laboratory parameters at the time of admission. RDW was measured as a part of the automated $\mathrm{CBC}$ panel. Automated analyzer Beckman Coulter LH 750 measured the CBC, and RDW was recorded as part of it. In our institution, $11.5 \%-14.5 \%$ is considered the reference range for $\mathrm{RDW}$-coefficient of variation $(\mathrm{CV})$. Patients found to be have anemia (as per WHO criteria) were further classified on the basis of RBC indices and peripheral blood smear into either of the following - microcytic (mean corpuscular volume $[\mathrm{MCV}]<80 \mathrm{fL}$ ), normocytic, or macrocytic $(\mathrm{MCV}>96 \mathrm{fL}) .{ }^{[17]}$ Iron profile was assessed for patients having microcytic and/or normocytic anemia. Vitamin B12 and folic acid levels were assessed for the patients having macrocytic anemia.

Primary outcome measure was 30-day mortality after admission. We chose to use 30-day mortality as it is an objective, clinically relevant, and well-accepted outcome indicator in studies pertaining to emergency and critical care area. It is less dependent on hospital discharge practices/policies. For those who died within 30 days during the hospital stay, the cause of death was documented from the case record files. Those who were discharged before 30 days, telephonic follow-up was done. This study was approved by thesis review committee. Informed consent was obtained from patient or next of kin.

\section{Statistical analysis}

Continuous data were expressed as mean \pm standard deviation (SD) or median and 25\%-75\% interquartile range, while categorical variables were presented as percentages $(\%)$ or proportions. Study population was divided into survivors and nonsurvivors; normal RDW group $(\leq 14.5 \%)$ and higher RDW group ( $>14.5 \%$ ). Between these groups, continuous and categorical variables were analyzed. Student's $t$-test was used to analyze normally distributed continuous variables, while Mann-Whitney U-test was used for nonnormally distributed continuous variables. Categorical variables were compared by means of Chi-square test. Variables with $P<0.1$ on univariate analysis for 30-day mortality were included in multivariate logistic regression analysis. The individual discriminatory values of RDW and APACHE II score for predicting 30 day mortality were studied using receiver operating characteristic (ROC) curve analyses with calculation of area under the curve (AUC).

Optimal cutoff value of RDW for predicting 30-day mortality was defined by calculating Youden's index. Youden's index is a value (derived from coordinates of ROC curve of RDW for 30-day mortality) at which the sum of sensitivity and specificity is maximum. Youden's index of RDW for predicting 30 -day mortality was calculated to be $17.3 \%$. Furthermore, the study population was subdivided into three groups using RDW cutoff values $14.5 \%$ (upper limit of normal reference range) and $17.3 \%$ (derived using Youden's index). Categorical variables between these groups were compared using Chi-square test. For normally distributed continuous variables between three groups, analysis of variance (ANOVA) test was used. Bonferroni's post hoc test was performed to evaluate the differences among RDW groups on finding significant $P$ values on ANOVA. For nonnormally distributed variables, Kruskal-Wallis test was used. For further characterization of significant $P$ values found on Kruskal-Wallis test, Mann-Whitney test was applied taking two out of three groups in different combinations. Data was recorded in the form of Excel worksheet. $P<0.05$ was considered statistically significant. All statistical tests performed were two-tailed. Statistical analysis was performed using SPSS 20.0 software (IBM Inc., New York, USA).

\section{RESULTS}

Two hundred and eleven patients were enrolled during the study period. Out of them, eleven patients had either missing data or were lost to follow-up and 200 patients were included in the final analysis. Mean age of study 
cohort was $51.32 \pm 16.98$ years (range $19-100$ years). Male preponderance (115 [57.5\%]) was noted. Most common site of sepsis at presentation was respiratory tract $(53.50 \%)$, followed by urinary tract (28\%), abdomen (21\%), skin and soft tissue $(9 \%)$, and central nervous system $(8.50 \%)$. One hundred and eighty-two patients $(91 \%)$ included in the study had community-acquired sepsis and the rest of the 18 patients ( $9 \%$ ) had hospital-acquired sepsis. Mean APACHE II score of study cohort at admission was $22.49 \pm 5.72$. Mean RDW at admission was $17.40 \pm 3.21 \%$ (range $12.60-33.30 \%$ ). One hundred and twenty-six patients $(63 \%)$ had anemia at admission (WHO classification). Among them, 114 patients had microcytic and/or normocytic anemia and 16 patients had macrocytic anemia. Fifty-two (52.5\%) out of 99 patients with iron profile who had microcytic and/or normocytic anemia were found to have iron deficiency. Iron profile could not be studied for 15 patients with microcytic anemia due to financial constraints. Mean duration of hospital stay was $10.00 \pm 9.15$ days [Tables 1 and 2].

Study population was divided into two groups based on RDW at admission - normal RDW group (RDW $\leq 14.5 \%)$ and raised RDW group (RDW $>14.5 \%$ ). Twenty patients $(10 \%)$ in the study population had normal RDW and 180 patients $(90 \%)$ had raised RDW at presentation. Demographic, clinical, and laboratory parameters were compared between these groups. Raised RDW group had significantly higher proportion of anemic patients (119/180 [66.11\%] vs. 7/20 [35.00\%], $P=0.006)$. Mean erythrocyte sedimentation rate (ESR) at baseline was higher in patient group with raised RDW although it did not reach the statistical significance. This patient group also had significantly higher proportion of patients with renal failure (136/180 [75.56\%] vs. 10/20 [50.00\%], $\mathrm{P}=0.031)$. Mean serum albumin at admission was significantly lower in high RDW group, while mean APACHE II score at admission was significantly higher in raised RDW group $(22.87 \pm 5.76$ vs. $19.05 \pm 3.95, P=0.004)$. 30-day mortality was significantly higher in raised RDW group $(108 / 180$ [60.0\%] vs. $6 / 20$ [30.00\%], $P=0.010)$. Further details of univariate analysis have been provided in Tables 3 and 4.

We further subdivided the study cohort into three groups using RDW values $14.5 \%$ (upper limit of normal range of RDW) and $17.3 \%$ (Youden's index, derived using coordinates of ROC curve of RDW for 30-day mortality): $\leq 14.5 \%$, $14.6 \%-17.3 \%$, and $>17.3 \%$. Across the three study groups, RDW at admission showed a significant graded association with mean hemoglobin, ESR, serum albumin, and bilirubin at baseline $(P<0.05)$. Moreover, RDW was also found to have significant graded association with APACHE II score at admission showing progressively increasing score along with rising RDW $(P<0.05)$. Thirty-day mortality showed a significant graded relationship with RDW at admission across these three groups. Thirty-day mortality rate was $30 \%$ in group of patients with RDW $\leq 14.5 \%, 53.4 \%$ in RDW $14.6 \%-17.3 \%$, and $68.8 \%$ in RDW $>17.3 \%(P=0.004)$ [Tables 5 and 6 ].

\begin{tabular}{|c|c|}
\hline Variables & $($ Mean \pm SD) \\
\hline Age (years) & $51.32 \pm 16.98$ \\
\hline Female/male & $85 / 115$ \\
\hline Anemia at admission, $n(\%)$ & $126(63.0)$ \\
\hline \multicolumn{2}{|l|}{ Source of sepsis, $n(\%)$} \\
\hline Community & $182(91.0)$ \\
\hline Hospital & $18(9.0)$ \\
\hline \multicolumn{2}{|l|}{ Comorbidities, $n(\%)$} \\
\hline Smoking & $73(36.5)$ \\
\hline Diabetes mellitus & $71(35.5)$ \\
\hline Alcoholic & $60(30)$ \\
\hline Cardiovascular disease & $27(13.5)$ \\
\hline Chronic kidney disease & $24(12.0)$ \\
\hline COPD & $21(10.5)$ \\
\hline Chronic liver disease & $13(6.5)$ \\
\hline Others & $8(4.0)$ \\
\hline \multicolumn{2}{|l|}{ Site of sepsis, $n(\%)$} \\
\hline Respiratory tract & $107(53.5)$ \\
\hline Urinary tract & $56(28)$ \\
\hline Abdominal infection & $42(21)$ \\
\hline Skin and soft tissue & $18(9)$ \\
\hline $\mathrm{CNS}$ & $17(8.5)$ \\
\hline GCS & $12.26 \pm 3.05$ \\
\hline BP systolic (mmHg) & $87.61 \pm 17.47$ \\
\hline BP diastolic (mmHg) & $55.86 \pm 11.59$ \\
\hline MAP (mmHg) & $66.16 \pm 13.20$ \\
\hline PR (beats/min) & $111.87 \pm 10.68$ \\
\hline RR (breaths/min) & $29.39 \pm 6.83$ \\
\hline \multicolumn{2}{|l|}{ Organ failure, $n(\%)$} \\
\hline Cardiovascular & $147(73.5)$ \\
\hline Renal & $146(73.0)$ \\
\hline Pulmonary & $123(61.5)$ \\
\hline $\mathrm{CNS}$ & $92(46.0)$ \\
\hline Hematologic & $64(32.0)$ \\
\hline Gastrointestinal & $45(22.5)$ \\
\hline \multicolumn{2}{|l|}{ Number of organ failures, $n(\%)$} \\
\hline 1 & $11(5.5)$ \\
\hline 2 & $55(27.5)$ \\
\hline 3 & $68(34.0)$ \\
\hline 4 & $42(21.0)$ \\
\hline 5 & $20(10.0)$ \\
\hline 6 & $4(2.0)$ \\
\hline APACHE II score at admission & $22.49 \pm 5.72$ \\
\hline EMOPD/EMW stay (days) & $5.60 \pm 3.51$ \\
\hline Hospital stay (days) & $10.00 \pm 9.15$ \\
\hline Vasoactive agent requirement, $n(\%)$ & $169(84.5)$ \\
\hline Renal replacement therapy, $n(\%)$ & $76(38.0)$ \\
\hline 30 days mortality, $n(\%)$ & $114(57)$ \\
\hline \multicolumn{2}{|c|}{$\begin{array}{l}\text { COPD: Chronic obstructive pulmonary disease; CNS: Central } \\
\text { nervous system; GCS: Glasgow coma scale; BP: Blood pressure; } \\
\text { MAP: Mean arterial pressure; PR: Pulse rate; RR: Respiratory rate; } \\
\text { APACHE II: Acute Physiology and Chronic Health Evaluation score; } \\
\text { EMOPD/EMW: Emergency medical outpatient department/emergency } \\
\text { medical ward; SD: Standard deviation }\end{array}$} \\
\hline
\end{tabular}




\begin{tabular}{|c|c|}
\hline Variables & (Mean \pm SD) \\
\hline Hemoglobin $(\mathrm{g} / \mathrm{dL})$ & $10.78 \pm 2.58$ \\
\hline Anaemia at admission, $n(\%)$ & $126(63.0)$ \\
\hline Iron deficiency, $n(\%)$ & $52 / 99(52.5)$ \\
\hline $\operatorname{TLC}(/ \mu \mathrm{L})$ & $22,859.20 \pm 10,309.93$ \\
\hline $\operatorname{ESR}\left(\mathrm{mm} 1^{\text {st }} \mathrm{h}\right)$ & $42.88 \pm 15.49$ \\
\hline Platelet $\left(\times 10^{3} / \mu \mathrm{L}\right)$ & $198.75 \pm 137.95$ \\
\hline Reticulocyte production index (\%) & $0.76 \pm 0.33$ \\
\hline Red cell distribution width (\%) & $17.40 \pm 3.21$ \\
\hline $\mathrm{MCV}(\mathrm{fL})$ & $86.11 \pm 8.72$ \\
\hline $\mathrm{MCH}(\mathrm{pg})$ & $28.34 \pm 3.13$ \\
\hline $\mathrm{MCHC}(\%)$ & $32.09 \pm 1.56$ \\
\hline Central venous $\mathrm{SpO}_{2}(\%)$ & $62.95 \pm 2.88$ \\
\hline Sodium $(\mathrm{mEq} / \mathrm{L})$ & $133.50 \pm 8.12$ \\
\hline Potassium $(\mathrm{mEq} / \mathrm{L})$ & $4.30 \pm 0.91$ \\
\hline Urea $(\mathrm{mg} / \mathrm{dL})$ & $113.92 \pm 80.56$ \\
\hline Creatinine (mg/dL) & $2.87 \pm 2.49$ \\
\hline SGOT (U/L), median (IQR) & $58.53(43.00-116.75)$ \\
\hline SGPT (U/L), median (IQR) & $26(31.00-72.88)$ \\
\hline ALP (U/L), median (IQR) & $131.00(108.00-176.50)$ \\
\hline Bilirubin (mg/dL), median (IQR) & $0.80(0.50-1.95)$ \\
\hline Total protein $(\mathrm{g} / \mathrm{dL})$ & $5.93 \pm 0.62$ \\
\hline Albumin $(\mathrm{g} / \mathrm{dL})$ & $3.07 \pm 0.48$ \\
\hline Globulin (g/dL) & $2.89 \pm 0.57$ \\
\hline RBS (mg/dL) & $149.63 \pm 68.42$ \\
\hline Calcium (mg/dL) & $8.77 \pm 0.81$ \\
\hline $\mathrm{pH}$ & $7.34 \pm 0.12$ \\
\hline $\mathrm{HCO}_{3}^{-}(\mathrm{mmol} / \mathrm{L})$ & $17.00 \pm 6.62$ \\
\hline $\mathrm{SpO}_{2}(\%)$ & $92.39 \pm 7.13$ \\
\hline $\mathrm{PaO}_{2} / \mathrm{FiO}_{2}$ & $234.32 \pm 82.49$ \\
\hline $\mathrm{PT}(\mathrm{s})$ & $22.21 \pm 11.32$ \\
\hline PT index $(\%)$ & $71.00 \pm 19.65$ \\
\hline aPTT (s) & $34.15 \pm 8.36$ \\
\hline Fibrinogen $(\mathrm{g} / \mathrm{dL})$ & $4.69 \pm 1.72$ \\
\hline Positive d-dimer, $n(\%)$ & $174(87)$ \\
\hline Lactate $(\mathrm{mmol} / \mathrm{L})(163)$ & $2.62 \pm 1.02$ \\
\hline \multicolumn{2}{|c|}{$\begin{array}{l}\text { TLC: Total leukocyte count; ESR: Erythrocyte sedimentation rate; } \\
\text { MCV: Mean corpuscular volume; MCH: Mean corpuscular hemoglobin; } \\
\text { MCHC: Mean corpuscular hemoglobin concentration; SGOT: Serum } \\
\text { glutamate oxaloacetate transaminase; SGPT: Serum glutamate pyruvate } \\
\text { transaminase; ALP: Alkaline phosphatase; } \mathrm{RBS} \text { : Random blood sugar; } \\
\mathrm{HCO}_{3}^{-} \text {: Bicarbonate; } \mathrm{SpO}_{2} \text { : Oxygen saturation; } \mathrm{PaO}_{2} / \mathrm{FiO}_{2} \text { ratio: Ratio } \\
\text { of partial pressure arterial oxygen and fraction of inspired oxygen; PT: } \\
\text { Prothrombin time; aPTT: Activated partial thromboplastin time; SD: } \\
\text { Standard deviation; IQR: Interquartile range }\end{array}$} \\
\hline
\end{tabular}

One hundred and fourteen (57\%) patients had 30-day mortality. Immediate cause of death in majority (110 patients [96.49\%]) was either refractory septic shock or progressive acute respiratory distress syndrome secondary to severe sepsis/ septic shock. Various parameters including demographic, clinical, laboratory and other variables such as organ dysfunction, severity of illness scores, and length of hospital stay were compared between survivors and nonsurvivors. Mean age, serum lactate, APACHE II score;

\begin{tabular}{|c|c|c|c|}
\hline \multirow{2}{*}{$\begin{array}{l}\text { Variables } \\
\text { (mean } \pm \text { SD) }\end{array}$} & \multicolumn{2}{|c|}{ RDW } & \multirow[t]{2}{*}{$P$} \\
\hline & $\leq 14.5(n=20)$ & $>14.5(n=180)$ & \\
\hline Age, (years) & $50.60 \pm 11.95$ & $51.40 \pm 17.48$ & 0.842 \\
\hline Female, $n(\%)$ & $7(35)$ & $78(43.33)$ & 0.474 \\
\hline Anemic, $n(\%)$ & $7(35.0)$ & $119(66.1)$ & 0.006 \\
\hline \multicolumn{4}{|l|}{ Source of sepsis, $n(\%)$} \\
\hline Community & $19(95.0)$ & $163(90.6)$ & 1.000 \\
\hline Hospital & $1(5.0)$ & $17(9.4)$ & 1.000 \\
\hline \multicolumn{4}{|l|}{ Site of sepsis, $n(\%)$} \\
\hline Respiratory tract & $9(45.0)$ & $98(54.4)$ & 0.422 \\
\hline Urinary tract & $5(25.0)$ & $51(28.3)$ & 1.000 \\
\hline Abdominal infection & $4(20.0)$ & $38(21.1)$ & 0.908 \\
\hline Skin and soft tissue & $1(5.0)$ & $17(9.4)$ & 1.000 \\
\hline $\mathrm{CNS}$ & $4(20.0)$ & $13(7.2)$ & 0.074 \\
\hline Alcoholic (60), $n(\%)$ & $8(40.0)$ & $52(28.9)$ & 0.312 \\
\hline Smoker (73), $n(\%)$ & $11(55.0)$ & $62(34.4)$ & 0.087 \\
\hline GCS & $12.35 \pm 3.00$ & $12.25 \pm 3.06$ & 0.890 \\
\hline BP systolic (mmHg) & $82.40 \pm 15.17$ & $88.19 \pm 17.64$ & 0.160 \\
\hline BP diastolic (mmHg) & $52.00 \pm 8.94$ & $56.29 \pm 11.79$ & 0.117 \\
\hline MAP (mmHg) & $62.05 \pm 10.62$ & $66.62 \pm 13.41$ & 0.142 \\
\hline PR (beats/min) & $113.80 \pm 9.75$ & $111.66 \pm 10.79$ & 0.396 \\
\hline RR (breaths/min) & $28.10 \pm 7.00$ & $29.53 \pm 6.82$ & 0.377 \\
\hline \multicolumn{4}{|l|}{ Organ failure, $n(\%)$} \\
\hline Cardiovascular (147) & $16(80.0)$ & $131(72.8)$ & 0.488 \\
\hline Renal (146) & $10(50.0)$ & $136(75.6)$ & 0.031 \\
\hline Pulmonary (123) & $13(65.0)$ & $110(61.1)$ & 0.735 \\
\hline CNS (92) & $7(35.0)$ & $85(47.2)$ & 0.354 \\
\hline Hematologic (64) & $7(35.0)$ & $57(31.7)$ & 0.762 \\
\hline Gastrointestinal (45) & $4(20.0)$ & $41(22.8)$ & 0.778 \\
\hline $\begin{array}{l}\text { APACHE II score at } \\
\text { admission }\end{array}$ & $19.05 \pm 3.95$ & $22.87 \pm 5.76$ & 0.004 \\
\hline $\begin{array}{l}\text { Days of mechanical } \\
\text { ventilation }\end{array}$ & $2.80 \pm 3.52$ & $3.29 \pm 3.65$ & 0.570 \\
\hline $\begin{array}{l}\text { EMOPD/EMW stay } \\
\text { (days) }\end{array}$ & $6.00 \pm 2.94$ & $5.55 \pm 3.57$ & 0.587 \\
\hline Hospital stay (days) & $9.95 \pm 9.47$ & $10.00 \pm 9.14$ & 0.984 \\
\hline \multicolumn{4}{|l|}{$\begin{array}{l}\text { Inotrope requirement, } \\
n(\%)\end{array}$} \\
\hline Yes (169) & $16(80.0)$ & $153(85.0)$ & 0.558 \\
\hline \multicolumn{4}{|l|}{$\begin{array}{l}\text { Renal replacement } \\
\text { therapy, } n(\%)\end{array}$} \\
\hline Yes $(76)$ & $3(15.0)$ & $73(40.6)$ & 0.025 \\
\hline 30-day mortality, $n(\%)$ & $6(30.0)$ & $108(60.0)$ & 0.010 \\
\hline $\begin{array}{l}\text { CNS: Central nervous } \\
\text { pressure; MAP: Mean a } \\
\text { rate; APACHE II: Acute } \\
\text { EMOPD/EMW: Emerg } \\
\text { medical ward; RWD: R }\end{array}$ & $\begin{array}{l}\text { em; GCS: Glasgo } \\
\text { ial pressure; PR: } \\
\text { lysiology and Ch }\end{array}$ & $\begin{array}{l}\text { coma scale; BP: Bl } \\
\text { lse rate; RR: Resp } \\
\text { ic Health Evaluati }\end{array}$ & $\begin{array}{l}\text { od } \\
\text { tory } \\
\text { l score; } \\
\text { ncy }\end{array}$ \\
\hline
\end{tabular}

proportion of patients with renal, central nervous system and gastrointestinal failure; proportion of patients requiring renal replacement therapy and vasoactive agents were significantly higher in 30-day nonsurvivors as compared to survivors. GCS, mean arterial pressure, serum albumin, $\mathrm{pH}$, ratio of 
Table 4: Comparison of various baseline laboratory parameters between study groups based on baseline red cell distribution width $\leq 14.5 \%$ and red cell distribution width $>14.5 \%$ in study population of 200 patients with severe sepsis

\begin{tabular}{|c|c|c|c|}
\hline \multirow[t]{2}{*}{ Variables (mean $\pm S D$ ) } & \multicolumn{2}{|c|}{ RDW } & \multirow[t]{2}{*}{$P$} \\
\hline & $\leq 14.5(n=20)$ & $>14.5(n=180)$ & \\
\hline Hemoglobin (g/dL) & $12.26 \pm 2.38$ & $10.62 \pm 2.56$ & 0.007 \\
\hline $\mathrm{TLC}(/ \mu \mathrm{L})$ & $22,495.00 \pm 9672.99$ & $22,899.67 \pm 10,403.03$ & 0.868 \\
\hline $\mathrm{ESR}\left(\mathrm{mm} 1^{\text {st }} \mathrm{h}\right)$ & $36.60 \pm 15.10$ & $43.57 \pm 15.42$ & 0.050 \\
\hline Platelet $\left(\times 10^{3} / \mu \mathrm{L}\right)$ & $194.45 \pm 144.22$ & $199.22 \pm 137.65$ & 0.884 \\
\hline Reticulocyte production index (\%) & $0.617 \pm 0.16$ & $0.777 \pm 0.34$ & 0.053 \\
\hline $\mathrm{MCV}(\mathrm{fL})$ & $89.03 \pm 7.00$ & $85.78 \pm 8.85$ & 0.115 \\
\hline $\mathrm{MCH}(\mathrm{pg})$ & $29.74 \pm 1.82$ & $28.19 \pm 3.21$ & 0.036 \\
\hline $\mathrm{MCHC}(\%)$ & $32.67 \pm 1.45$ & $32.03 \pm 1.56$ & 0.081 \\
\hline Central venous $\mathrm{SpO}_{2}(\%)$ & $63.59 \pm 3.01$ & $62.88 \pm 2.87$ & 0.310 \\
\hline Sodium $(\mathrm{mEq} / \mathrm{L})$ & $132.52 \pm 8.12$ & $133.64 \pm 8.11$ & 0.518 \\
\hline Potassium $(\mathrm{mEq} / \mathrm{L})$ & $4.25 \pm 0.82$ & $4.34 \pm 1.04$ & 0.751 \\
\hline Chloride (mEq/L) & $96.94 \pm 8.67$ & $98.27 \pm 9.14$ & 0.537 \\
\hline Urea $(\mathrm{mg} / \mathrm{dL})$ & $77.55 \pm 43.02$ & $118.00 \pm 82.79$ & 0.033 \\
\hline Creatinine $(\mathrm{mg} / \mathrm{dL})$ & $1.85 \pm 1.29$ & $3.00 \pm 2.56$ & 0.055 \\
\hline SGOT (U/L), median (IQR) & $66.65(34.50-141.00)$ & $58.00(43.00-111.75$ & 0.751 \\
\hline SGPT (U/L), median (IQR) & $48.80(30.25-132.08)$ & $39.00(31.00-72.13)$ & 0.484 \\
\hline ALP (U/L), median (IQR) & $121.00(90.50-155.75)$ & $132.00(108.50-177.75)$ & 0.182 \\
\hline Bilirubin $(\mathrm{mg} / \mathrm{dL})$, median $(\mathrm{IQR})$ & $0.80(0.50-2.08)$ & $0.80(0.50-1.95)$ & 0.866 \\
\hline Total protein $(\mathrm{g} / \mathrm{dL})$ & $6.00 \pm 0.59$ & $5.93 \pm 0.62$ & 0.740 \\
\hline Albumin $(\mathrm{g} / \mathrm{dL})$ & $3.29 \pm 0.57$ & $3.05 \pm 0.47$ & 0.028 \\
\hline Globulin (g/dL) & $2.79 \pm 0.62$ & $2.89 \pm 0.57$ & 0.460 \\
\hline $\mathrm{RBS}(\mathrm{mg} / \mathrm{dL})$ & $157.80 \pm 69.08$ & $148.72 \pm 68.47$ & 0.575 \\
\hline Calcium (mg/dL) & $8.58 \pm 0.59$ & $8.79 \pm 0.83$ & 0.255 \\
\hline Magnesium (mg/dL) & $1.97 \pm 0.47$ & $1.92 \pm 0.45$ & 0.624 \\
\hline $\mathrm{pH}$ & $7.37 \pm 0.10$ & $7.33 \pm 0.12$ & 0.164 \\
\hline $\mathrm{PaO}_{2}(\mathrm{mmHg})$ & $82.72 \pm 28.78$ & $86.36 \pm 30.76$ & 0.614 \\
\hline $\mathrm{PaCO}_{2}(\mathrm{mmHg})$ & $29.53 \pm 8.56$ & $31.00 \pm 12.41$ & 0.610 \\
\hline $\mathrm{HCO}_{3}^{-}(\mathrm{mmol} / \mathrm{L})$ & $18.00 \pm 5.76$ & $16.85 \pm 6.71$ & 0.477 \\
\hline $\mathrm{SpO}_{2}(\%)$ & $93.30 \pm 5.89$ & $92.29 \pm 7.26$ & 0.556 \\
\hline $\mathrm{PaO}_{2} / \mathrm{FiO}_{2}$ ratio & $255.41 \pm 89.00$ & $231.89 \pm 81.66$ & 0.227 \\
\hline $\mathrm{PT}(\mathrm{s})$ & $23.45 \pm 17.76$ & $22.07 \pm 10.43$ & 0.605 \\
\hline PTI (\%) & $75.40 \pm 25.00$ & $70.50 \pm 19.00$ & 0.291 \\
\hline aPTT (s) & $31.45 \pm 8.73$ & $34.45 \pm 8.31$ & 0.129 \\
\hline Fibrinogen $(\mathrm{g} / \mathrm{dL})$ & $4.93 \pm 1.32$ & $4.67 \pm 1.75$ & 0.514 \\
\hline \multicolumn{4}{|l|}{ d-dimer } \\
\hline Positive, $n(\%)$ & $18(90.0)$ & $156(86.7)$ & 0.674 \\
\hline Lactate $(\mathrm{mmol} / \mathrm{L})(163)$ & $2.68 \pm 1.13$ & $2.61 \pm 1.01$ & 0.788 \\
\hline
\end{tabular}

partial pressure arterial oxygen $\left(\mathrm{PaO}_{2}\right)$ fraction of inspired oxygen $\left(\mathrm{FiO}_{2}\right)\left(\mathrm{PaO}_{2} / \mathrm{FiO}_{2}\right.$ ratio $)$, mean central venous oxygen saturation, serum fibrinogen at admission, and duration of hospital stay were significantly lower in nonsurvivors as compared to survivors. Survivors and nonsurvivors groups had no statistical difference in the proportion of patients with anemia at admission. Nonsurvivors during the hospital stay developed significantly lower platelet counts. Mean RDW at admission in nonsurvivors (17.84 $\pm 3.38 \%)$ was significantly higher than survivors $(16.84 \pm 2.90 \%)$
$(P=0.029)$ [Tables 7 and 8 ]. In multivariate logistic regression analyses, however, APACHE II score at admission, serum albumin, $\mathrm{PaO}_{2} / \mathrm{FiO}_{2}$ ratio, and serum fibrinogen at admission were found to be independent predictors of 30-day mortality $(P<0.05)$ [Table 9 ]. To assess the diagnostic accuracy for predicting 30-day mortality, ROC curve analysis was done and AUC was calculated. AUC for APACHE II score at admission was 0.822 (95\% confidence interval [CI], 0.764-0.880), $(\mathrm{P}<0.001)$; and AUC for RDW at admission was $0.606(95 \% \mathrm{CI}, 0.527-0.685)(P<0.001)$. 


\begin{tabular}{|c|c|c|c|c|}
\hline Variables (mean $\pm S D$ ) & $\begin{array}{c}\text { Group I (RDW } \leq 14.5) \\
(n=20)\end{array}$ & $\begin{array}{l}\text { Group II (RDW 14.6-17.3) } \\
\qquad(n=103)\end{array}$ & $\begin{array}{l}\text { Group III (RDW >17.3) } \\
\qquad(n=77)\end{array}$ & $P$ \\
\hline Age (years) & $50.60 \pm 11.95$ & $52.54 \pm 18.24$ & $49.87 \pm 16.38$ & 0.570 \\
\hline Female/male & $7 / 13$ & $41 / 62$ & $37 / 40$ & 0.419 \\
\hline Anemia at admission (126), $n(\%)$ & $7(35.0)$ & $56(44.4)$ & $63(50.0)$ & $<0.001^{\mathrm{a}, \mathrm{c}}$ \\
\hline \multicolumn{5}{|l|}{ Site of sepsis, $n(\%)$} \\
\hline Respiratory tract (107) & $9(45.0)$ & $56(54.4)$ & $42(54.5)$ & 0.724 \\
\hline Urinary tract $(56)$ & $5(25.0)$ & $34(33.1)$ & $17(22.1)$ & 0.258 \\
\hline Abdominal infection (42) & $4(20.0)$ & $20(19.4)$ & $18(23.4)$ & 0.807 \\
\hline Skin and soft tissue (18) & $1(5.0)$ & $5(4.9)$ & $12(15.6)$ & $0.036^{\mathrm{b}, \mathrm{c}}$ \\
\hline CNS (17) & $4(20)$ & $8(7.8)$ & $5(6.5)$ & 0.144 \\
\hline Alcoholic $(60), n(\%)$ & $8(40.0)$ & $29(28.2)$ & $23(29.9)$ & 0.571 \\
\hline Smoker (73), $n(\%)$ & $11(55.0)$ & $38(36.9)$ & $24(31.2)$ & 0.142 \\
\hline GCS & $12.35 \pm 3.00$ & $12.24 \pm 3.19$ & $12.26 \pm 2.89$ & 0.990 \\
\hline BP systolic (mmHg) & $82.40 \pm 15.17$ & $87.26 \pm 17.76$ & $89.43 \pm 17.52$ & 0.266 \\
\hline BP diastolic (mmHg) & $52.00 \pm 8.94$ & $56.23 \pm 11.79$ & $56.36 \pm 11.88$ & 0.293 \\
\hline MAP (mmHg) & $62.05 \pm 10.62$ & $66.12 \pm 13.65$ & $67.33 \pm 13.13$ & 0.281 \\
\hline PR (beats/min) & $113.80 \pm 9.75$ & $110.87 \pm 9.32$ & $112.70 \pm 12.48$ & 0.367 \\
\hline RR (breaths/min) & $28.10 \pm 7.00$ & $29.25 \pm 6.17$ & $29.90 \pm 7.64$ & 0.558 \\
\hline \multicolumn{5}{|l|}{ Organ failure, $n(\%)$} \\
\hline Cardiovascular (147) & $16(80.0)$ & $74(71.8)$ & $57(74.0)$ & 0.745 \\
\hline Renal (146) & $10(50.0)$ & $74(71.8)$ & $62(80.5)$ & 0.022 \\
\hline Pulmonary (123) & $13(65.0)$ & $61(59.2)$ & $49(63.6)$ & 0.788 \\
\hline CNS (92) & $7(35.0)$ & $48(46.6)$ & $37(48.1)$ & 0.571 \\
\hline Hematologic (64) & $7(35.0)$ & $32(31.1)$ & $25(32.5)$ & 0.936 \\
\hline Gastrointestinal (45) & $4(20.0)$ & $23(22.3)$ & $18(23.4)$ & 0.948 \\
\hline APACHE II score at admission & $19.05 \pm 3.95$ & $22.23 \pm 5.64$ & $23.73 \pm 5.85$ & $0.003^{\mathrm{a}, \mathrm{c}}$ \\
\hline Days of mechanical ventilation & $2.80 \pm 3.52$ & $3.10 \pm 4.08$ & $3.55 \pm 4.00$ & 0.611 \\
\hline EMOPD/EMW stay (days) & $6.00 \pm 2.94$ & $5.53 \pm 3.47$ & $5.80 \pm 3.72$ & 0.861 \\
\hline Hospital stay (days) & $9.95 \pm 9.47$ & $9.13 \pm 8.18$ & $11.16 \pm 10.23$ & 0.340 \\
\hline \multicolumn{5}{|l|}{ Inotrope requirement, $n(\%)$} \\
\hline Yes $(169)$ & $16(8)$ & $83(80.6)$ & $70(90.9)$ & 0.140 \\
\hline \multicolumn{5}{|l|}{ Renal replacement therapy, $n(\%)$} \\
\hline Yes $(76)$ & $3(15.0)$ & $35(33.9)$ & $38(49.4)$ & $0.009^{c}$ \\
\hline 30-day mortality & $6(30.0)$ & $55(53.4)$ & $53(68.8)$ & $0.004^{c}$ \\
\hline
\end{tabular}

\section{Discussion}

In this prospective observational study, we evaluated the role of RDW as a prognostic marker of 30-day mortality in patients with severe sepsis presenting in emergency services of a large referral hospital. RDW was significantly associated with 30-day mortality in patients with severe sepsis across all age groups and a variety of comorbidities in univariate analysis; however, it was not found to be an independent predictor of 30-day mortality. At admission, APACHE II score, serum albumin, $\mathrm{PaO}_{2} / \mathrm{FiO}_{2}$ ratio, and serum fibrinogen were observed to be independent predictors of 30-day mortality in this cohort of severe sepsis patients.

$\mathrm{RDW}$ is a measure of degree of RBC size variability. It is calculated as the SD in RBC size divided by the MCV. RDW values are determined by many factors. Elevation in RDW has been shown to be associated primarily with conditions that lead to ineffective production or increased destruction of RBC. Sepsis syndrome influences erythropoiesis through various mechanisms. Elevated inflammatory markers (tumor necrosis factor $\alpha$, interleukin 6 , interleukin $1 \beta$, etc.,) affect the RBC survival and maturation. Early release of immature, larger RBCs into the circulation results in elevated RDW. Pro-inflammatory state in sepsis syndrome also leads to decreased erythropoietin production, resistance to its effect, as well as decreased iron bioavailability. Erythroid precursor activity is thus suppressed in the bone marrow. ${ }^{[12,18-20]}$ Oxidative stress may also be a contributor for RDW-mortality association in sepsis. Elevated RDW is seen in states of high oxidative stress. It occurs by decreased RBC survival and release of large premature RBCs into circulation. ${ }^{[21]}$ 
Table 6: Comparison of various baseline laboratory parameters according to three groups of red cell distribution width at admission in study population of 200 patients with severe sepsis

\begin{tabular}{|c|c|c|c|c|}
\hline Variables (mean $\pm S D$ ) & $\begin{array}{c}\text { Group I (RDW } \leq 14.5) \\
(n=20)\end{array}$ & $\begin{array}{l}\text { Group II (RDW 14.6-17.3) } \\
\qquad(n=103)\end{array}$ & $\begin{array}{l}\text { Group III (RDW >17.3) } \\
(n=77)\end{array}$ & $P^{\#}$ \\
\hline Hemoglobin (g/dL) & $12.26 \pm 2.38$ & $11.39 \pm 2.45$ & $9.58 \pm 2.32$ & $<0.001^{\mathrm{b}, \mathrm{c}}$ \\
\hline Iron deficiency, $n(\%)$ & $1(5.0)$ & $17(18.5)$ & $34(46.6)$ & $<0.001$ \\
\hline $\operatorname{TLC}(/ \mu \mathrm{L})$ & $22495.00 \pm 9672.99$ & $22333.01 \pm 9668.04$ & $23657.66 \pm 11332.63$ & 0.688 \\
\hline ESR (mm 1st h) & $36.60 \pm 15.10$ & $40.36 \pm 15.18$ & $47.87 \pm 14.76$ & $0.001^{\mathrm{b}, \mathrm{c}}$ \\
\hline Platelet $\left(\times 10^{3} / \mu \mathrm{L}\right)$ & $194.45 \pm 144.22$ & $195.42 \pm 126.50$ & $204.31 \pm 152.00$ & 0.904 \\
\hline Reticulocyte production index (\%) & $0.78 \pm 0.34$ & $0.72 \pm 0.30$ & $0.85 \pm 0.38$ & $0.011^{\mathrm{b}, \mathrm{c}}$ \\
\hline $\mathrm{MCV}(\mathrm{fL})$ & $89.03 \pm 7.00$ & $85.82 \pm 7.37$ & $85.74 \pm 10.55$ & 0.289 \\
\hline $\mathrm{MCH}(\mathrm{pg})$ & $29.74 \pm 1.82$ & $28.44 \pm 2.90$ & $27.84 \pm 3.57$ & $0.027^{\mathrm{c}}$ \\
\hline Sodium $(\mathrm{mEq} / \mathrm{L})$ & $132.51 \pm 8.12$ & $134.29 \pm 8.52$ & $132.77 \pm 7.49$ & 0.033 \\
\hline Potassium $(\mathrm{mEq} / \mathrm{L})$ & $4.23 \pm 0.84$ & $4.28 \pm 0.86$ & $4.27 \pm 1.05$ & 0.011 \\
\hline Urea $(\mathrm{mg} / \mathrm{dL})$ & $77.55 \pm 43.02$ & $112.83 \pm 79.85$ & $124.82 \pm 86.61$ & 0.063 \\
\hline Creatinine (mg/dL) & $1.85 \pm 1.29$ & $2.75 \pm 2.28$ & $3.29 \pm 2.89$ & 0.056 \\
\hline SGOT (U/L), median (IQR) & $66.65(34.50-141.00)$ & $54.00(41.60-131.00)$ & $61.00(43.50-92.20)$ & 0.846 \\
\hline SGPT (U/L), median (IQR) & $48.80(30.25-132.08)$ & $39.00(31.00-79.10)$ & $39.00(28.10-71.75)$ & 0.626 \\
\hline Bilirubin $(\mathrm{mg} / \mathrm{dL})$, median (IQR) & $0.80(0.50-2.08)$ & $0.70(0.40-1.40)$ & $1.00(0.60-2.90)$ & $0.031 \mathrm{~b}$ \\
\hline Total protein $(\mathrm{g} / \mathrm{dL})$ & $6.00 \pm 0.59$ & $5.91 \pm 0.63$ & $5.95 \pm 0.61$ & 0.894 \\
\hline Albumin $(\mathrm{g} / \mathrm{dL})$ & $3.29 \pm 0.57$ & $3.13 \pm 0.49$ & $2.94 \pm 0.43$ & $0.002^{\mathrm{b}, \mathrm{c}}$ \\
\hline Globulin (g/dL) & $2.79 \pm 0.62$ & $2.79 \pm 0.55$ & $3.03 \pm 0.57$ & $0.012^{\mathrm{b}}$ \\
\hline $\mathrm{RBS}(\mathrm{mg} / \mathrm{dL})$ & $157.80 \pm 69.08$ & $150.05 \pm 68.86$ & $146.94 \pm 68.36$ & 0.817 \\
\hline Calcium (mg/dL) & $8.58 \pm 0.59$ & $8.82 \pm 0.71$ & $8.76 \pm 1.00$ & 0.472 \\
\hline $\mathrm{pH}$ & $7.37 \pm 0.10$ & $7.34 \pm 0.10$ & $7.32 \pm 0.14$ & 0.176 \\
\hline $\mathrm{PaO}_{2} / \mathrm{FiO}_{2}$ & $255.41 \pm 89.01$ & $239.48 \pm 85.07$ & $221.72 \pm 76.21$ & 0.174 \\
\hline $\mathrm{PT}(\mathrm{s})$ & $23.45 \pm 17.77$ & $21.86 \pm 11.34$ & $22.34 \pm 9.14$ & 0.843 \\
\hline PTI (\%) & $75.40 \pm 25.00$ & $71.74 \pm 19.00$ & $68.84 \pm 19.04$ & 0.356 \\
\hline aPTT (s) & $31.45 \pm 8.73$ & $33.83 \pm 7.75$ & $35.29 \pm 9.00$ & 0.162 \\
\hline Fibrinogen $(\mathrm{g} / \mathrm{dL})$ & $4.93 \pm 1.32$ & $4.86 \pm 1.73$ & $4.40 \pm 1.82$ & 0.151 \\
\hline Lactate $(\mathrm{mmol} / \mathrm{L})(163)$ & $2.68 \pm 1.13$ & $2.41 \pm 0.87$ & $2.86 \pm 1.11$ & $0.047^{\mathrm{b}}$ \\
\hline
\end{tabular}

Raised RDW group had significantly higher percentage of patients with anemia and renal dysfunction. Serum albumin was also lower in this group. We noted on dividing study population further into three groups - RDW $\leq 14.5 \%, 14.6 \%-17.3 \%$, and $>17.3 \%$, a graded association was seen between RDW and mean hemoglobin, ESR, serum albumin, bilirubin and lactate at baseline. In response to infection/sepsis, liver responds to inflammatory state by increasing production of number of acute-phase reactants, e.g., ESR/CRP, and concurrently, production of so-called "negative" acute-phase reactants such as serum albumin is reduced. Relationship of these two markers with RDW might be indirectly reflective of degree of inflammation in index study as well. However, anemia and concurrent renal dysfunction might have contributed to elevated RDW in present study.

In index study, the primary outcome, i.e., 30-day mortality, was $57.0 \%$. Jo et al. reported $29.0 \%$ mortality at the end of 28-day follow-up while studying the prognostic value of RDW in severe sepsis and septic shock. ${ }^{[14]}$ A prospective, observational, multicenter study was undertaken by Lorente et al. in six Spanish Intensive Care Units. Of 297 severe sepsis patients recruited, 104 (35.01\%) had 30-day mortality. ${ }^{[22]} \mathrm{RDW}$ also showed a hierarchical association with 30-day mortality among three RDW groups in index study. But in multivariate logistic regression, APACHE II score, serum albumin, $\mathrm{PaO}_{2} / \mathrm{FiO}_{2}$ ratio, and serum fibrinogen level at admission were independent predictors of 30-day mortality. In early eighties, sepsis was thought to be driven primarily by microorganism. Later concept of host driven pro-inflammatory response took primacy which was reflected in "SIRS" definition of sepsis. Later research revealed that sepsis elicits a very complex, fluctuating, and prolonged host response. Results from various studies reveal rapid release of both pro-inflammatory and anti-inflammatory cytokines in some patients with sepsis. ${ }^{[2]}$ Many patients on the other hand have either markedly raised quantity of anti-inflammatory cytokines, or there is generalized depression in cytokine production. This variable immune response is modulated by the load and virulence of causative pathogens, genetic characteristics, and comorbid illnesses of 
Table 7: Comparison of demographic and clinical parameters between survivors and nonsurvivors in study population of 200 patients with severe sepsis

\begin{tabular}{|c|c|c|c|}
\hline Variables (mean $\pm S D$ ) & Survivors $(n=86)$ & Nonsurvivors $(n=114)$ & $P$ \\
\hline Age (years) & $47.57 \pm 17.13$ & $54.15 \pm 16.38$ & 0.006 \\
\hline Female/male & $34 / 52$ & $51 / 63$ & 0.461 \\
\hline Anemia at admission, $n(\%)$ & $51(59.3)$ & $75(65.8)$ & 0.347 \\
\hline \multicolumn{4}{|l|}{ Source of sepsis, $n(\%)$} \\
\hline Community & $80(93)$ & $102(89.5)$ & 0.460 \\
\hline Hospital & $6(7)$ & $12(10.5)$ & 0.460 \\
\hline \multicolumn{4}{|l|}{ Comorbidities, $n(\%)$} \\
\hline Diabetes mellitus (71) & $27(31.4)$ & $44(38.6)$ & 0.292 \\
\hline Cardiovascular disease (27) & $7(8.1)$ & $20(17.5)$ & 0.054 \\
\hline Chronic kidney disease (24) & $7(8.1)$ & $17(14.9)$ & 0.145 \\
\hline Chronic liver disease (13) & $4(4.7)$ & $9(7.9)$ & 0.357 \\
\hline Chronic obstructive pulmonary disease (21) & $5(5.8)$ & $16(14.0)$ & 0.060 \\
\hline Alcoholic (60) & $24(27.9)$ & $36(31.5)$ & 0.575 \\
\hline Smoker (73) & $28(32.6)$ & $45(39.5)$ & 0.315 \\
\hline Others $(8)$ & $3(3.5)$ & $5(4.4)$ & 0.748 \\
\hline \multicolumn{4}{|l|}{ Site of sepsis, $n(\%)$} \\
\hline Respiratory tract (107) & $43(50)$ & $64(56.1)$ & 0.389 \\
\hline Urinary tract (56) & $22(25.6)$ & $34(29.8)$ & 0.508 \\
\hline Abdominal infection (42) & $18(20.9)$ & $24(21.0)$ & 0.983 \\
\hline Skin and soft tissue (18) & $8(9.3)$ & $10(8.8)$ & 1.000 \\
\hline CNS (17) & $8(9.3)$ & $9(7.9)$ & 0.897 \\
\hline GCS at admission & $13.52 \pm 2.39$ & $11.31 \pm 3.15$ & $<0.001$ \\
\hline BP systolic (mmHg) & $91.47 \pm 16.61$ & $84.70 \pm 17.59$ & 0.001 \\
\hline BP diastolic (mmHg) & $58.02 \pm 10.46$ & $54.23 \pm 12.18$ & 0.009 \\
\hline MAP (mmHg) & $69.13 \pm 12.07$ & $63.93 \pm 13.62$ & 0.001 \\
\hline PR (beats/min) & $111.42 \pm 10.24$ & $112.21 \pm 11.04$ & 0.577 \\
\hline RR (breaths/min) & $29.34 \pm 7.13$ & $29.42 \pm 6.63$ & 0.932 \\
\hline \multicolumn{4}{|l|}{ Organ failure, $n(\%)$} \\
\hline Cardiovascular (147) & $58(67.4)$ & $89(78.1)$ & 0.107 \\
\hline Renal (146) & $55(63.9)$ & $91(79.8)$ & 0.012 \\
\hline Pulmonary (123) & $49(57.0)$ & $74(64.9)$ & 0.304 \\
\hline CNS (92) & $20(23.3)$ & $72(63.2)$ & $<0.001$ \\
\hline Hematologic (64) & $24(27.9)$ & $40(35.1)$ & 0.289 \\
\hline Gastrointestinal (45) & $13(15.1)$ & $32(28.1)$ & 0.040 \\
\hline APACHE II score at admission & $18.93 \pm 4.18$ & $25.18 \pm 5.25$ & $<0.001$ \\
\hline Days of mechanical ventilation & $1.7 \pm 3.1$ & $4.3 \pm 3.5$ & $<0.001$ \\
\hline EMOPD/EMW stay (days) & $5.67 \pm 4.39$ & $5.54 \pm 2.87$ & 0.782 \\
\hline Hospital stay (days) & $13.42 \pm 12.00$ & $7.40 \pm 4.84$ & $<0.001$ \\
\hline \multicolumn{4}{|l|}{ Vasoactive agent requirement, $\mathrm{n}(\%)$} \\
\hline Yes $(169)$ & $59(68.6)$ & $110(96.5)$ & $<0.001$ \\
\hline \multicolumn{4}{|l|}{ Renal replacement therapy, n (\%) } \\
\hline Yes $(76)$ & $25(29.1)$ & $51(44.7)$ & 0.024 \\
\hline
\end{tabular}

host. ${ }^{[23,24]}$ Severity of illness scores are summative manifestation of these various pathophysiological processes in sepsis and have been validated in studies as best prognostic indicators. APACHE and SOFA are the scores validated as prognostic factor in various studies. ${ }^{[25]}$ Data from 5815 patients across 13 hospitals from the United States contributed to the development of APACHE II score. Many physiologic variables, age, previous health status, and admission diagnosis were used in this severity of illness model. ${ }^{[26]}$ In index study as well, on multivariate analysis, admission APACHE II score was one of the independent prognostic markers. Significantly lower mean fibrinogen level among nonsurvivors can possibly be explained by development of disseminated intravascular coagulation as supported by higher prothrombin time (PT) as well as activated partial thromboplastin time values and lower platelet counts among nonsurvivors during the hospital stay. Higher incidence 
Table 8: Comparison of various baseline laboratory parameters between survivors and nonsurvivors in study population of 200 patients with severe sepsis

\begin{tabular}{|c|c|c|c|}
\hline Baseline laboratory parameters (mean \pm SD) & Survivors $(n=86)$ & Nonsurvivors $(n=114)$ & $P$ \\
\hline Hemoglobin $(\mathrm{g} / \mathrm{dL})$ & $10.90 \pm 2.77$ & $10.68 \pm 2.43$ & 0.565 \\
\hline Anemia at admission, $n(\%)$ & $51(59.3)$ & $75(65.8)$ & 0.347 \\
\hline Iron deficiency $(99), n(\%)$ & $25(31.6)$ & $27(25.5)$ & 0.355 \\
\hline $\operatorname{TLC}(/ \mu \mathrm{L})$ & $22,178.37 \pm 8743.31$ & $23,372.81 \pm 11,360.87$ & 0.419 \\
\hline $\operatorname{ESR}\left(\mathrm{mm}^{\mathrm{st}} \mathrm{h}\right)$ & $43.55 \pm 14.04$ & $42.37 \pm 16.55$ & 0.596 \\
\hline Platelet $\left(\times 10^{3} / \mu \mathrm{L}\right)$ & $196.07 \pm 136.72$ & $200.76 \pm 139.45$ & 0.812 \\
\hline Reticulocyte production index (\%) & $0.71 \pm 0.29$ & $0.79 \pm 0.36$ & 0.074 \\
\hline Red cell distribution width (\%) & $16.84 \pm 2.90$ & $17.84 \pm 3.38$ & 0.029 \\
\hline $\mathrm{MCV}(\mathrm{fL})$ & $85.09 \pm 9.46$ & $86.87 \pm 8.06$ & 0.154 \\
\hline $\mathrm{MCH}(\mathrm{pg})$ & $27.89 \pm 3.27$ & $28.68 \pm 3.00$ & 0.077 \\
\hline $\mathrm{MCHC}(\%)$ & $32.07 \pm 1.43$ & $32.11 \pm 1.65$ & 0.884 \\
\hline Central venous $\mathrm{SpO}_{2}(\%)$ & $63.77 \pm 3.09$ & $62.37 \pm 2.58$ & 0.001 \\
\hline Sodium $(\mathrm{mEq} / \mathrm{L})$ & $131.49 \pm 13.60$ & $134.17 \pm 8.08$ & 0.084 \\
\hline Potassium (mEq/L) & $4.25 \pm 0.84$ & $4.34 \pm 1.01$ & 0.242 \\
\hline Urea $(\mathrm{mg} / \mathrm{dL})$ & $104.50 \pm 76.59$ & $121.03 \pm 83.05$ & 0.151 \\
\hline Creatinine (mg/dL) & $2.69 \pm 2.59$ & $3.01 \pm 2.41$ & 0.406 \\
\hline SGOT (U/L), median (IQR) & $54.00(43.00-84.03)$ & $61.95(40.50-149.69)$ & 0.470 \\
\hline SGPT (U/L), median (IQR) & $38.00(32.00-63.50)$ & 43.45 (29.00-79.52) & 0.602 \\
\hline ALP (U/L), median (IQR) & $137.00(109.00-181.50)$ & $130.50(108.00-166.00)$ & 0.737 \\
\hline Bilirubin (mg/dL), median (IQR) & $0.80(0.50-1.50)$ & $0.80(0.50-2.50)$ & 0.891 \\
\hline Total protein $(\mathrm{g} / \mathrm{dL})$ & $6.01 \pm 0.49$ & $5.91 \pm 0.69$ & 0.118 \\
\hline Albumin $(\mathrm{g} / \mathrm{dL})$ & $3.22 \pm 0.48$ & $3.00 \pm 0.46$ & $<0.001$ \\
\hline Globulin (g/dL) & $2.82 \pm 0.43$ & $2.94 \pm 0.66$ & 0.135 \\
\hline RBS (mg/dL) & $146.88 \pm 66.01$ & $151.69 \pm 70.39$ & 0.624 \\
\hline Calcium (mg/dL) & $8.74 \pm 0.79$ & $8.79 \pm 0.81$ & 0.601 \\
\hline $\mathrm{pH}$ & $7.36 \pm 0.10$ & $7.31 \pm 0.13$ & 0.004 \\
\hline $\mathrm{HCO}_{3}^{-}(\mathrm{mmol} / \mathrm{L})$ & $17.42 \pm 5.43$ & $16.62 \pm 7.39$ & 0.403 \\
\hline $\mathrm{SpO}_{2}(\%)$ & $93.59 \pm 4.20$ & $91.48 \pm 8.62$ & 0.039 \\
\hline $\mathrm{PaO}_{2} / \mathrm{FiO}_{2}$ ratio & $251.72 \pm 78.08$ & $221.04 \pm 83.59$ & 0.003 \\
\hline $\mathrm{PT}(\mathrm{s})$ & $20.70 \pm 7.39$ & $23.34 \pm 13.47$ & 0.102 \\
\hline PTI (\%) & $73.00 \pm 17.95$ & $69.50 \pm 20.80$ & 0.218 \\
\hline aPTT (s) & $33.08 \pm 6.01$ & $35.00 \pm 9.74$ & 0.117 \\
\hline Fibrinogen $(\mathrm{g} / \mathrm{dL})$ & $5.36 \pm 1.47$ & $4.19 \pm 1.72$ & $<0.001$ \\
\hline Positive d-dimer (174), $n(\%)$ & $74(42.5)$ & $100(57.5)$ & 0.728 \\
\hline Lactate $(\mathrm{mmol} / \mathrm{L})(163)$ & $2.31 \pm 1.00$ & $2.83 \pm 1.00$ & $<0.001$ \\
\hline
\end{tabular}

TLC: Total leucocyte count; ESR: Erythrocyte sedimentation rate; MCV: Mean corpuscular volume; MCH: Mean corpuscular hemoglobin; MCHC: Mean corpuscular hemoglobin concentration; SGOT: Serum glutamate oxaloacetate transaminase; SGPT: Serum glutamate pyruvate transaminase; ALP: Alkaline phosphatase; RBS: Random blood sugar; $\mathrm{HCO}_{3}^{-}$: Bicarbonate; $\mathrm{SpO}_{2}$ : Oxygen saturation; $\mathrm{PaO}_{2} / \mathrm{FiO}_{2}$ : Ratio of partial pressure arterial oxygen and fraction of inspired oxygen; PT: Prothrombin time; PTI: Prothrombin time index; aPTT: Activated partial thromboplastin time; IQR: Inter-quartile range; SD: Standard deviation

of liver dysfunction in nonsurvivors can be another possible explanation. $\mathrm{PaO}_{2} / \mathrm{FiO}_{2}$ ratio is a measure of oxygenation status and is one of the criteria for diagnosis of acute respiratory distress syndrome in adults. In index study, most common admitting diagnosis was pulmonary infection. Serum albumin has been proved to be a robust prognostic indicator in various studies. Several mechanisms might help explain this apparent relationship. Serum albumin plays important role in binding and transport of important biomolecules. It maintains the colloid osmotic pressure and has got free radical scavenging activity. It has antithrombotic properties and has effects on vascular permeability. ${ }^{[27]}$ A meta-analysis involving 90 cohort studies with 291,433 total patients evaluated prognostic significance of hypoalbuminemia. Authors found significant, dose-dependent independent association of hypoalbuminemia with worse outcome. This association was independent of both inflammation and nutritional status. ${ }^{[28]} \mathrm{RDW}$, though indicator of many adverse processes, was not independently associated with 30-day mortality. As stated earlier, sepsis is a complex disease process, not just a pro-inflammatory state. Treatment interventions directed specifically at controlling the pro-inflammatory state have failed miserably in various studies emphasizing the complexity of pathologic process in sepsis. ${ }^{[29]}$ Severity of illness/organ dysfunction scores are indicative of cumulative effect of these various complex pathophysiological processes in sepsis. Understandably, prognostic value of these parameters would be greater as compared to RDW, which is corroborated with our study results. ROC curve analyses also 


\begin{tabular}{|c|c|c|c|c|}
\hline \multicolumn{5}{|c|}{$\begin{array}{l}\text { Table 9: Adjusted multivariate logistic regression analysis } \\
\text { for } 30 \text { days mortality in study population of } 200 \text { patients } \\
\text { with severe sepsis }\end{array}$} \\
\hline Variable & $\begin{array}{l}\text { Regression } \\
\text { coefficient }\end{array}$ & OR & $95 \% \mathrm{Cl}$ & $P$ \\
\hline $\begin{array}{l}\text { APACHE II score } \\
\text { at admission }\end{array}$ & 0.226 & 1.254 & $1.151-1.366$ & $<0.001$ \\
\hline $\begin{array}{l}\text { Serum albumin at } \\
\text { admission }\end{array}$ & -0.986 & 0.373 & 0.151-0.919 & 0.032 \\
\hline $\begin{array}{l}\mathrm{PaO}_{2} / \mathrm{FiO}_{2} \text { ratio } \\
\text { at admission }\end{array}$ & -0.006 & 0.994 & 0.989-0.999 & 0.014 \\
\hline $\begin{array}{l}\text { Serum fibrinogen } \\
\text { at admission }\end{array}$ & -0.294 & 0.745 & $0.577-0.962$ & 0.024 \\
\hline
\end{tabular}

APACHE II: Acute Physiology and Chronic Health Evaluation score; $\mathrm{PaO}_{2} / \mathrm{FIO}_{2}$ : Ratio of partial pressure arterial oxygen and fraction of inspired oxygen; OR: Odds ratio; CI: Confidence intervals

revealed marginal discriminatory power of RDW (AUC 0.606) for predicting 30-day mortality as compared to APACHE II score (AUC 0.822).

This study had a prospective design. All patients were managed under similar settings with uniform management protocol based on Surviving Sepsis Guidelines. We included patients having a wide range of comorbidities and variety of illnesses. There was uniformity in the time of measurement of RDW, i.e., at admission in emergency medical services. Hence, baseline RDW was not affected by medical management during hospitalization. By reviewing transfusion records before admission, patients with history of transfusion of blood products were not included. Blood transfusion is an important confounder for raised RDW. Because of their retrospective design, earlier studies did not address this important aspect. The primary outcome of the study, i.e., 30-day mortality, is an objective, clinically relevant, and well-accepted outcome in studies pertaining to critical care.

This study also had certain limitations. Being single-center study and conducted in a large tertiary care institution, results may not be generalizable to other health care institutions. As it included only patients admitted to emergency medical services, results may not be extrapolated to the ward and surgical patients with severe sepsis. This study included 200 patients, a sample size which may not seem as robust as earlier studies. This study did not include estimation of inflammatory cytokine levels.

\section{Conclusions}

RDW was not found to be an independent predictor of 30-day mortality. At admission, APACHE II score, serum albumin, $\mathrm{PaO}_{2} / \mathrm{FiO}_{2}$ ratio, and serum fibrinogen level were observed to be independent predictors of 30-day mortality in cohort of severe sepsis patients admitted to emergency medical services.

\section{Financial support and sponsorship}

Nil.

\section{Conflicts of interest}

There are no conflicts of interest.

\section{RefEREnCes}

1. Mayr FB, Yende S, Angus DC. Epidemiology of severe sepsis. Virulence 2014;5:4-11.

2. Angus DC, van der Poll T. Severe sepsis and septic shock. N Engl J Med 2013;369:840-51.

3. Bone RC, Sibbald WJ, Sprung CL. The ACCP-SCCM consensus conference on sepsis and organ failure. Chest 1992;101:1481-3.

4. Levy MM, Fink MP, Marshall JC, Abraham E, Angus D, Cook D, et al. $2001 \mathrm{SCCM} / \mathrm{ESICM} / \mathrm{ACCP} / \mathrm{ATS} / \mathrm{SIS}$ International Sepsis Definitions Conference. Crit Care Med 2003;31:1250-6.

5. Afessa B, Keegan MT, Mohammad Z, Finkielman JD, Peters SG. Identifying potentially ineffective care in the sickest critically ill patients on the third ICU day. Chest 2004;126:1905-9.

6. Annane D, Bellissant E, Cavaillon JM. Septic shock. Lancet 2005;365:63-78.

7. Marshall JC, Reinhart K, International Sepsis Forum. Biomarkers of sepsis. Crit Care Med 2009;37:2290-8.

8. Hou H, Sun T, Li C, Li Y, Guo Z, Wang W, et al. An overall and dose-response meta-analysis of red blood cell distribution width and CVD outcomes. Sci Rep 2017;7:43420.

9. Zorlu A, Bektasoglu G, Guven FM, Dogan OT, Gucuk E, Ege MR, et al. Usefulness of admission red cell distribution width as a predictor of early mortality in patients with acute pulmonary embolism. Am J Cardiol 2012;109:128-34.

10. Braun E, Domany E, Kenig Y, Mazor Y, Makhoul BF, Azzam ZS, et al. Elevated red cell distribution width predicts poor outcome in young patients with community acquired pneumonia. Crit Care 2011;15:R194.

11. Bazick HS, Chang D, Mahadevappa K, Gibbons FK, Christopher KB. Red cell distribution width and all-cause mortality in critically ill patients. Crit Care Med 2011;39:1913-21.

12. Pierce $\mathrm{CN}$, Larson DF. Inflammatory cytokine inhibition of erythropoiesis in patients implanted with a mechanical circulatory assist device. Perfusion 2005;20:83-90.

13. Lippi G, Targher G, Montagnana M, Salvagno GL, Zoppini G, Guidi GC, et al. Relation between red blood cell distribution width and inflammatory biomarkers in a large cohort of unselected outpatients. Arch Pathol Lab Med 2009;133:628-32.

14. Jo YH, Kim K, Lee JH, Kang C, Kim T, Park HM, et al. Red cell distribution width is a prognostic factor in severe sepsis and septic shock. Am J Emerg Med 2013;31:545-8.

15. Wang F, Pan W, Pan S, Ge J, Wang S, Chen M, et al. Red cell distribution width as a novel predictor of mortality in ICU patients. Ann Med 2011;43:40-6.

16. Kim CH, Park JT, Kim EJ, Han JH, Han JS, Choi JY, et al. An increase in red blood cell distribution width from baseline predicts mortality in patients with severe sepsis or septic shock. Crit Care 2013;17:R282.

17. World Health Organization. Haemoglobin Concentrations for the Diagnosis of Anaemia and Assessment of Severity. Vitamin and Mineral Nutrition Information System Geneva: WHO; 2011. Available from: http://www.who.int/vmnis/indicators/haemoglobin.pdf. [Last accessed on 2017 May 02].

18. Salvagno GL, Sanchis-Gomar F, Picanza A, Lippi G. Red blood cell distribution width: A simple parameter with multiple clinical applications. Crit Rev Clin Lab Sci 2015;52:86-105.

19. Scharte M, Fink MP. Red blood cell physiology in critical illness. Crit Care Med 2003;31:S651-7.

20. Weiss G, Goodnough LT. Anemia of chronic disease. N Engl J Med 2005;352:1011-23.

21. Kolls JK. Oxidative stress in sepsis: A redox redux. J Clin Invest 2006;116:860-3.

22. Lorente L, Martín MM, Abreu-González P, Solé-Violán J, Ferreres J, Labarta L, et al. Red blood cell distribution width during the first week is associated with severity and mortality in septic patients. PLoS One 2014;9:e105436.

23. van der Poll T, van de Veerdonk FL, Scicluna BP, Netea MG. The immunopathology of sepsis and potential therapeutic targets. Nat Rev Immunol 2017;17:407-20.

24. Hotchkiss RS, Monneret G, Payen D. Immunosuppression in sepsis: 
A novel understanding of the disorder and a new therapeutic approach. Lancet Infect Dis 2013;13:260-8.

25. Afessa B, Gajic O, Keegan MT. Severity of illness and organ failure assessment in adult intensive care units. Crit Care Clin 2007;23:639-58.

26. Knaus WA, Draper EA, Wagner DP, Zimmerman JE. APACHE II: Aseverity of disease classification system. Crit Care Med 1985;13:818-29.

27. Fanali G, di Masi A, Trezza V, Marino M, Fasano M, Ascenzi P, et al.
Human serum albumin: From bench to bedside. Mol Aspects Med 2012;33:209-90.

28. Vincent JL, Dubois MJ, Navickis RJ, Wilkes MM. Hypoalbuminemia in acute illness: Is there a rationale for intervention? A meta-analysis of cohort studies and controlled trials. Ann Surg 2003;237:319-34.

29. Hotchkiss RS, Sherwood ER. Immunology. Getting sepsis therapy right. Science 2015;347:1201-2 